Original Article

\title{
Chronic Angiotensin II Inhibition Increases Levels of Calcitonin Gene-Related Peptide mRNA of the Dorsal Root Ganglia in Spontaneously Hypertensive Rats
}

\author{
Hiromu KAWASAKI, Keiichi INAIZUMI, Arisa NAKAMURA, \\ Narumi HOBARA, and Yuji KUROSAKI
}

\begin{abstract}
We previously reported that the vasodilation mediated by calcitonin gene-related peptide (CGRP)-containing nerves and level of CGRP mRNA in the dorsal root ganglia (DRG) in spontaneously hypertensive rats (SHR) decreased with age, and that the reduced function of CGRP nerves was restored by chronic inhibition of angiotensin II. The present study was performed to investigate the effect of long-term treatment with angiotensin II type-1 receptor antagonists (L-158,809 and olmesartan), an angiotensin converting enzyme inhibitor (temocapril) and hydralazine on levels of CGRP mRNA in DRG of SHR and the contents of CGRP in the mesenteric artery and atrium. The level of CGRP mRNA and degree of CGRP-like immunoreactivities (CGRP-LI) were measured by Northern blot hybridization assay and enzyme-linked immunosorbent assay, respectively. Seven week-treatment of 8 week-old SHR with temocapril $(0.005 \%)$, L-158,809 (0.001\%), olmesartan $(0.01 \%)$ or hydralazine $(0.01 \%)$ administered in drinking water significantly lowered the systolic blood pressure of SHR. The level of CGRP mRNA in the DRG of control SHR was significantly lower than that in normotensive Wistar Kyoto rats (WKY), whereas the level of CGRP-LI in the mesenteric artery and atrium of SHR were significantly greater than those in WKY. Treatment of SHR with temocapril, L-158,809, or olmesartan, but not hydralazine, significantly elevated the levels of CGRP mRNA in DRG, markedly increased the level of CGRP-LI in the mesenteric artery, and slightly increased the CGRP-LI level in the atrium. These results suggest that long-term inhibition of angiotensin II restores the reduced expression of CGRP mRNA in DRG and may facilitate neurotransmission of CGRP-containing vasodilator nerves in SHR.
\end{abstract}

(Hypertens Res 2003; 26: 257-263)

Key Words: angiotensin II type-1 receptor antagonist, calcitonin gene-related peptide (CGRP)-containing vasodilator nerve, CGRP mRNA, tissue CGRP contents, spontaneously hypertensive rat

\section{Introduction}

Non-adrenergic non-cholinergic vasodilator nerves innervate the resistance arteries and play a role in the regulation of vascular tone $(1,2)$. Previous studies have demonstrated that the rat mesenteric resistance artery is innervated by nonadrenergic non-cholinergic vasodilator nerves in which calcitonin gene-related peptide (CGRP), a potent vasodilator neuropeptide, acts as the vasodilator neurotransmitter $(1,3$, 4). CGRP is distributed throughout the central and peripheral nervous systems and is located in areas involved in cardio-

From the Department of Clinical Pharmaceutical Science, Graduate School of Natural Science and Technology, Okayama University, Okayama, Japan.

This study was supported by Grants-in-Aid for Scientific Research (KAKENHI) from the Ministry of Education, Culture, Sports Science, and Technology of Japan (Nos. 09672326 and 10557244).

Address for Reprints: Hiromu Kawasaki, Ph.D., Department of Clinical Pharmaceutical Science, Graduate School of Natural Science and Technology, Okayama University, 1-1-1 Tsushima-naka, Okayama 700-8530, Japan. E-mail: kawasaki@pheasant.pharm.okayama-u.ac.jp

Received August 8, 2002; Accepted in revised form November 13, 2002. 
vascular function (5). The dorsal root ganglia (DRG) is a prominent site of CGRP synthesis and contains the cell bodies of primary afferent neurons that extend CGRP-containing (CGRPergic) nerves to peripheral sites such as the blood vessels and the central spinal cord (6). We have reported that CGRPergic nerves suppress vasoconstriction in response to sympathetic adrenergic nerve stimulation via CGRP release, and conversely, adrenergic nerves inhibit the release of CGRP from the nerve to decrease CGRPergic nerve function $(3,4)$. Thus, we have proposed that CGRPergic vasodilator nerves along with sympathetic vasoconstrictor nerves regulate the tone of the mesenteric resistance artery.

Impairment of the control systems regulating peripheral resistance has been considered to play a role in chronic hypertension, and increased total peripheral vascular resistance has been shown to contribute to the maintenance of elevated blood pressure (7-10). Studies with spontaneously hypertensive rats (SHR) have shown that the enhanced activity of sympathetic vasoconstrictor nerves contributes to the increased tone of peripheral resistance arteries (9-11). Previous studies have demonstrated that both the vasodilation mediated by CGRPergic nerves and the release of CGRP from the nerves in the mesenteric artery of SHR are smaller than those in normotensive Wistar Kyoto rats (WKY), and that these differences decrease with age (12). In recent studies, we have shown that the levels of CGRP mRNA in DRG of 15-week-old SHR were lower than those in WKY, and this phenomenon was also age-related (13). Therefore, we proposed that malfunction of CGRPergic vasodilator nerves regulating peripheral vascular resistance plays an important role in the development and maintenance of hypertension in SHR (12-14). However, the mechanisms underlying the reduced function of CGRPergic vasodilator nerves in SHR remain unresolved. In previous pharmacological studies, we showed that angiotensin II inhibits neurotransmission of CGRPergic nerves in SHR (15), and that chronic treatment of SHR with angiotensin converting enzyme (ACE) inhibitors-but not treatment with a calcium antagonist, $\beta$-adrenoceptor antagonist, or other antihypertensive drugs - prevents the decrease in vasodilator responses mediated by CGRPergic nerves $(16,17)$.

The present study was, therefore, undertaken to investigate the effect of long-term treatment of SHR with an ACE inhibitor (temocapril) and selective angiotensin II type-1 receptor $\left(\mathrm{AT}_{1} \mathrm{R}\right)$ antagonists, 2-ethyl-5,7-dimethyl-3-[[2' $-(1 \mathrm{H}-$ tetrazol-5-yl) [1,1'-biphenyl]-4-yl] methyl]-3H-imidazol [4,5B] pyridine (L-158,809) (18) and olmesartan (19), on the levels of CGRP mRNA in DRG and the tissue contents of CGRP.

\section{Methods}

\section{Animals}

Male SHR and WKY at 8 weeks of age (purchased from Shimizu Experimental Animal, Shizuoka, Japan) were used for this study. The animals were given food and water $a d l i$ - bitum. They were housed in the Animal Research Center of Okayama University at a controlled ambient temperature of $22^{\circ} \mathrm{C}$ with $50 \pm 10 \%$ relative humidity and with a 12-h light/12-h dark cycle (lights on at 8:00 AM). This study was performed in accordance with the Guide for Animal Experimentation of the Faculty of Pharmaceutical Science, Okayama University.

\section{Long-Term Treatment with Antihypertensive Drugs}

Male SHR at 8 weeks of age received $0.005 \%$ temocapril ( $5 \mathrm{mg} / \mathrm{kg} / \mathrm{day}$ ), $0.001 \%$ L-158,809 ( $1 \mathrm{mg} / \mathrm{kg} /$ day), $0.01 \%$ olmesartan $(10 \mathrm{mg} / \mathrm{kg} /$ day $)$ or $0.01 \%$ hydralazine $(10 \mathrm{mg} / \mathrm{kg}$ /day) in their drinking water and normal rat chow. Each SHR received the respective treatment for a period of 7 weeks. Control SHR and WKY received normal rat chow and drinking water supplement with $0.1 \% \mathrm{NaHCO}_{3}$ solution, which was used as vehicle for temocapril, L-158,809, olmesartan and hydralazine.

\section{Systemic Blood Pressure Measurement}

The systolic blood pressure of each animal was measured weekly by tail-cuff plethysmography (model TK-370C; UNICOM, Tokyo, Japan) throughout the treatment period.

\section{Measurement of CGRP mRNA Levels}

At 15 weeks of age, all animals were deeply anesthetized with a large dose of pentobarbital- $\mathrm{Na}(50 \mathrm{mg} / \mathrm{kg}$ i.p. $)$ and killed by decapitation. The thoracic and lumber DRG, kidney and thyroid gland from each rat were immediately dissected, frozen in liquid nitrogen, and stored at $-80^{\circ} \mathrm{C}$ until subsequent analysis of CGRP mRNA.

Each tissue was homogenized in $4 \mathrm{~mol} / \mathrm{l}$ guanidine thiocyanate, $0.5 \%$ sodium- $\mathrm{N}$-lauril sarcosine, $25 \mathrm{mmol} / \mathrm{l}$ sodium citrate, and $0.1 \mathrm{~mol} / \mathrm{l}$ 2-mercaptoethanol, and total cellular RNA was extracted with phenol/chloroform (20). The RNA was then precipitated once in the same volume of isopropyl alcohol and once in 2.5 volumes of ethanol. Thereafter, the precipitated RNA was washed in $75 \%$ ethanol and dissolved in water. The amount of RNA was quantified by absorbance at $260 \mathrm{~nm}$ in a spectrophotometer. The RNA samples were denatured by heating at $65^{\circ} \mathrm{C}$ for $15 \mathrm{~min}$, and the denatured RNA was electrophoresed in $1 \%$ agarose gel at $90 \mathrm{~V}$ for $3 \mathrm{~h}$. The RNA was then capillary transferred onto a nylon membrane (Gene Screen Plus; NEN Life Science Products, Inc., Boston, USA) with $10 \times$ standard saline citrate (SSC) $(1 \times$ SSC: $0.15 \mathrm{~mol} / 1$ sodium chloride, $15 \mathrm{mmol} / \mathrm{l}$ sodium citrate, $\mathrm{pH}$ 7.0) for $4 \mathrm{~h}$. The RNA on the nylon membrane was fixed using UV irradiation. The CGRP cDNA and glyceraldehyde3-phosphate dehydrogenase (GAPDH) cDNA were labeled with $\left[\alpha-{ }^{32} \mathrm{P}\right] \mathrm{dCTP}(\sim 3,000 \mathrm{Ci} / \mathrm{mmol}$; NEN Life Science Products) using Megaprime DNA labeling systems (Amersham Int. Plc., Buckinghamshire, UK). After the nylon mem- 


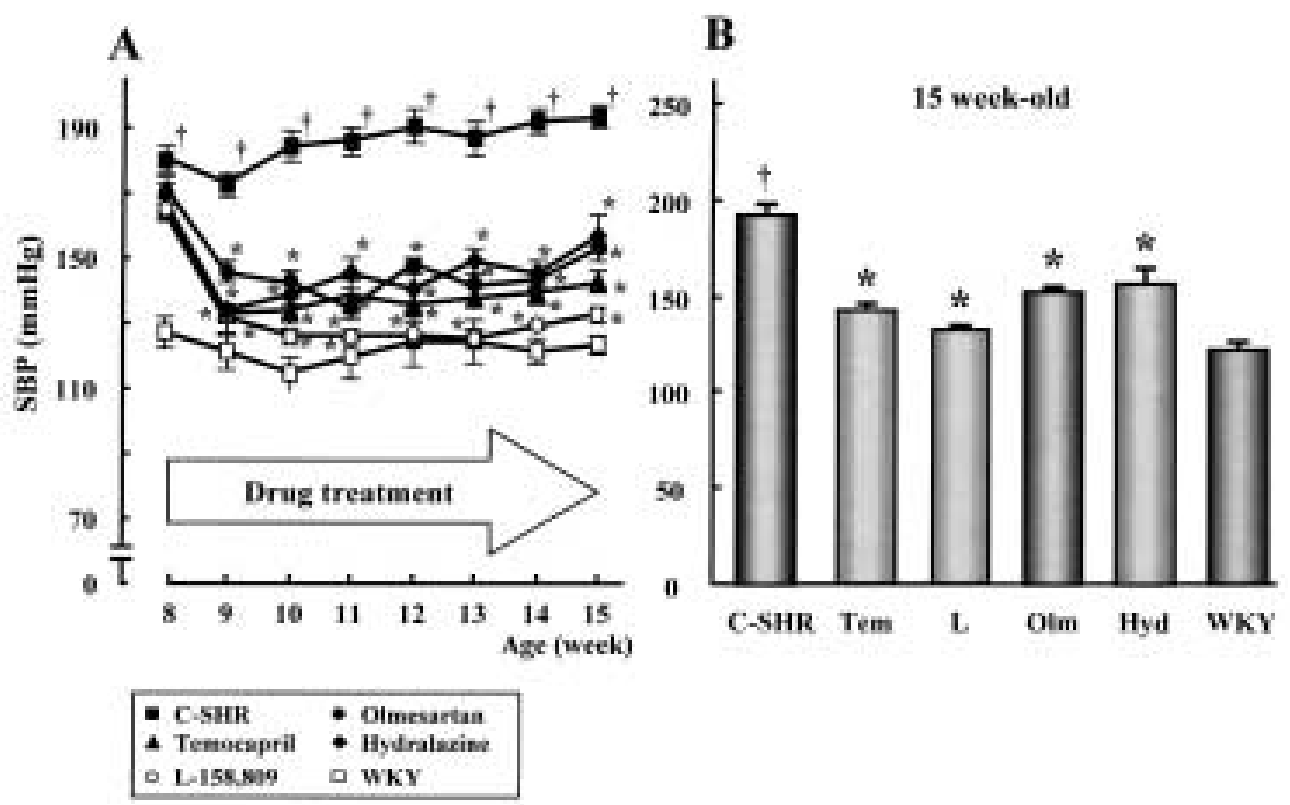

Fig. 1. Line graph (A) showing changes in systolic blood pressure (SBP) of spontaneously hypertensive rats (SHR) during 7 weeks of treatment with various antihypertensive drugs and bar graph (B) showing SBP in 15-week-old SHR following 7 weeks of drug treatment. C-SHR, control SHR; Tem, temocapril; L, L-158,809; Olm, olmesartan; Hyd, hydralazine; WKY, normotensive Wistar Kyoto rats. Each bar indicates the mean \pm SEM of 6 experiments. ${ }^{*} \mathrm{p}<0.01$, compared with control SHR. ${ }^{\dagger} \mathrm{p}<0.01$, compared with WKY.

brane was prehybridized with Rapid-Hyb buffer (Amersham Int. Plc.) for $15 \mathrm{~min}$ at $65^{\circ} \mathrm{C}$, the ${ }^{32} \mathrm{p}$-labeled probe denatured by heating at $95^{\circ} \mathrm{C}$ for $5 \mathrm{~min}$ was added to the buffer. The nylon membrane was then hybridized for $2 \mathrm{~h}$ at $65^{\circ} \mathrm{C}$. After this procedure, the blotted nylon membrane was washed twice at $65^{\circ} \mathrm{C}$ in a solution containing $2 \times \operatorname{SSPE}(0.3 \mathrm{~mol} / \mathrm{l}$ $\mathrm{NaCl}, 17.3 \mathrm{mmol} / \mathrm{l} \mathrm{NaH}_{2} \mathrm{PO}_{4}, 2.5 \mathrm{mmol} / \mathrm{l}$ EDTA, pH 7.4), and $0.1 \%$ sodium dodecyl sulfate (SDS) for $10 \mathrm{~min}$, twice in a solution containing $1 \times$ SSPE and $0.1 \%$ SDS for $20 \mathrm{~min}$, and then once in a solution containing $0.1 \times$ SSPE and $0.1 \%$ SDS at $65^{\circ} \mathrm{C}$ for $20 \mathrm{~min}$. Autoradiography was performed at room temperature using FUJI Imaging Plates (FUJI Photo Film Co., Tokyo, Japan). The radioactivity of each band was quantified by means of computerized densitometiric scanning (BAS 2000; Fuji Photo Film Co.). All results were expressed relative to the amount of GAPDH mRNA, which served as a control for the quality and quantify of RNA applied to the blot.

\section{Measurement of Tissue CGRP Contents}

The atrium and mesenteric artery isolated from control SHR, WKY and drug-treated SHR at 15 weeks of age were homogenized and centrifuged at 7,500 rpm. The supernatant was applied to a Bond-Elut C18 cartridge (Varian, Harbor city, USA), and the absorbed peptide was eluted with $60 \%$ acetonitrile in $1 \%$ trifluoroacetic acid. The elute was evaporated under a vacuum and the levels of CGRP-like immunoreactivities (CGRP-LI) were determined using a commercially available enzyme-linked immunosorbent assay (ELISA) for CGRP kit (Peninsula Laboratories Inc., San Carlos, USA).

\section{Statistical Analysis}

Values are presented as the means \pm SEM. One-way analysis of variance followed by Tukey's test was used to determine the significance between values of different drugs. Unpaired Student's $t$-test was used to determine the significance of differences between two means. Values of $p<0.05$ were considered to indicate statistical significance.

\section{Drugs}

The following drugs were used: hydralazine hydrochloride (Sigma Chemical Co., St. Louis, USA), L-158,809 (Merck \& Co., Inc., Rahway, USA), olmesartan medoxomil (Sankyo Co, Tokyo, Japan), temocapril (Sankyo Co.). Olmesartan, L158,809 , temocapril and hydralazine were dissolved in drinking water containing $0.1 \% \mathrm{NaHCO}_{3}$ at final concentrations of $0.01 \%, 0.001 \%, 0.005 \%$ and $0.01 \%$, respectively.

\section{Results}

\section{Changes in Systolic Blood Pressure during Long- Term Treatment with Antihypertensive Drugs}

As shown in Fig. 1, long-term treatment with olmesartan, L- 


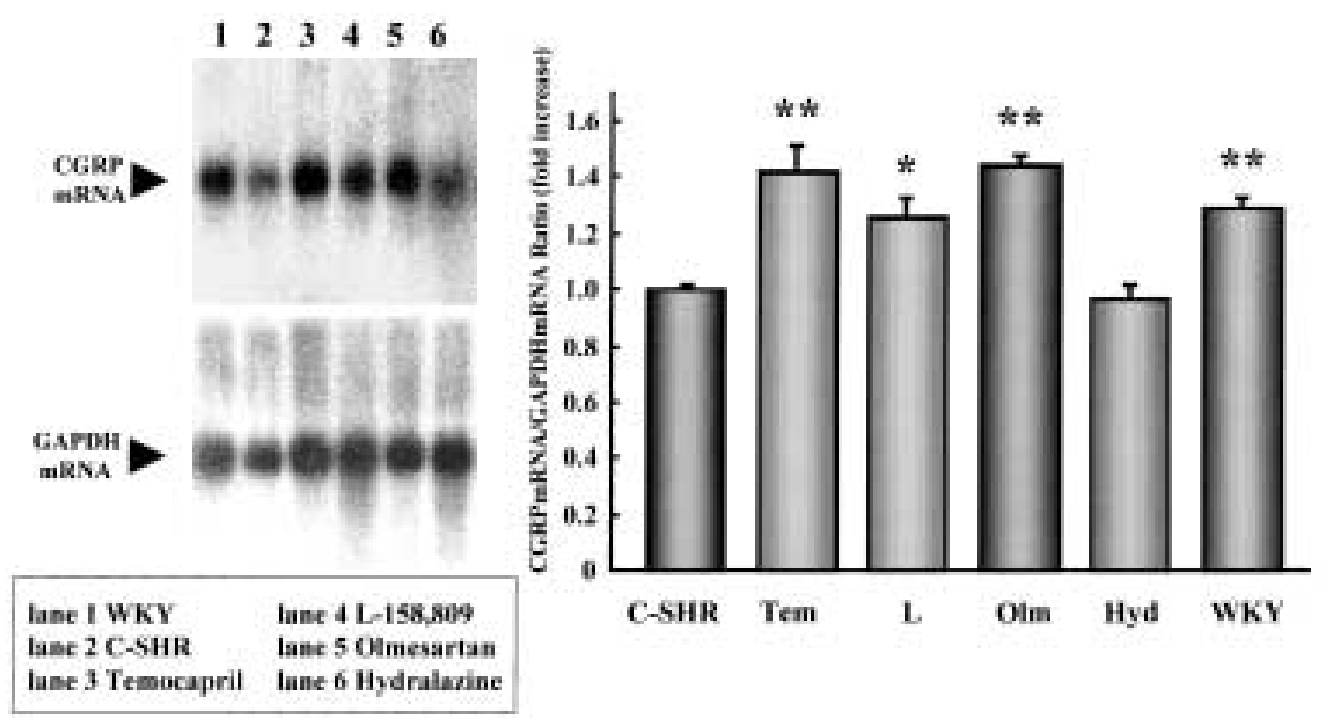

Fig. 2. Effect of long-term treatment with antihypertensive drugs on calcitonin gene-related peptide (CGRP) mRNA expression in the dorsal root ganglia (DRG) of spontaneously hypertensive rats (SHR). A: Representative image of Northern blot assays of CGRP $m R N A$ and glyceraldehyde-3-phosphate dehydrogenase (GAPDH) mRNA. B: The ratio of CGRP $m R N A / G A P D H$ mRNA determined by densitometric analysis of Northern blot assays in the DRG of control SHR, drug-treated SHR and WKY. Lanes 1 to 6 show WKY, control SHR, temocapril, L-158,809, olmesartan and hydralazine, respectively. C-SHR, control SHR; Tem, temocapril; L, L-158,809; Olm, olmesartan; Hyd, hydralazine; WKY, normotensive Wistar Kyoto rats. Each bar indicates the mean \pm SEM of 6 experiments and fold-increase over the control SHR. ${ }^{*} \mathrm{p}<0.05,{ }^{* *} \mathrm{p}<0.01$, compared with control SHR.
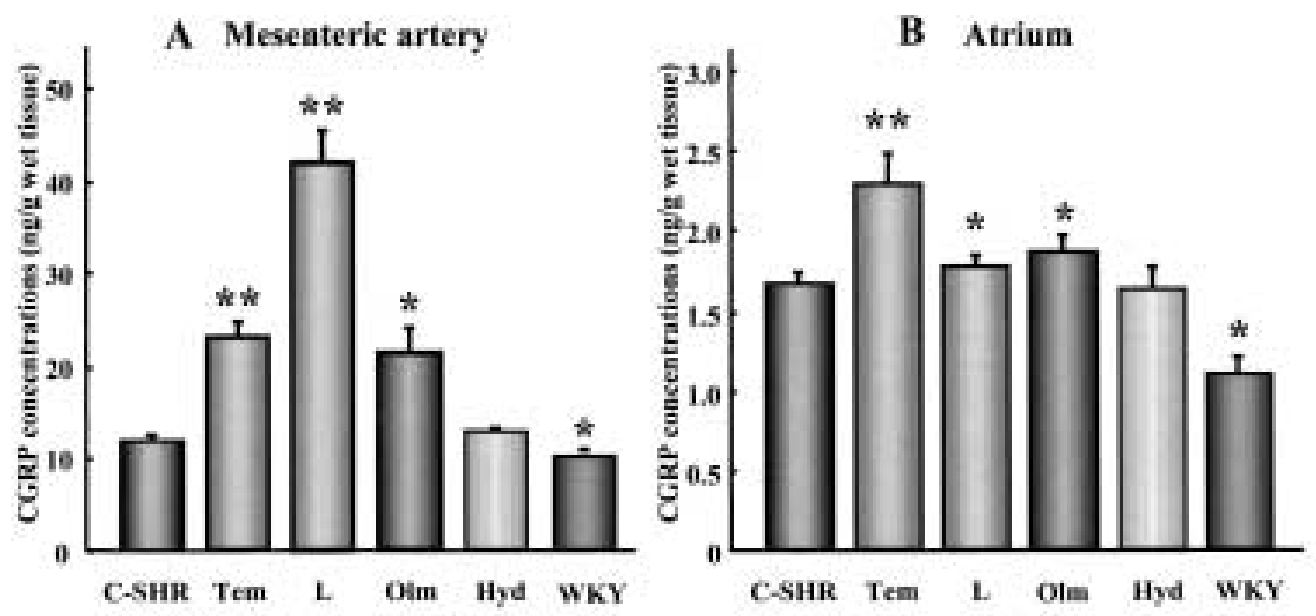

Fig. 3. Effect of long-term treatment with various antihypertensive drugs on levels of calcitonin gene-related peptide-like immunoreactivities (CGRP-LI) in mesenteric arteries (A) and atria (B) of spontaneously hypertensive rats (SHR). C-SHR, control SHR; Tem, temocapril; L, L-158,809; Olm, olmesartan; Hyd, hydralazine; WKY, normotensive Wistar Kyoto rats. Each bar indicates the mean \pm SEM of 5 experiments. ${ }^{*} \mathrm{p}<0.05,{ }^{* *} \mathrm{p}<0.01$, compared with control SHR.

158,809 , temocapril and hydralazine significantly lowered systolic blood pressure in SHR, compared with that in control SHR. Significant differences were found between nontreated SHR and antihypertensive drug-treated SHR. Significant differences were also found between control SHR and WKY.

\section{Changes in CGRP mRNA Levels}

Figure 2A shows a representative Northern blot analysis of CGRP mRNA. High levels of CGRP mRNA were apparent in the DRG of both WKY and SHR. A small amount of CGRP mRNA was observed in the thyroid gland, but little or no CGRP mRNA was found in the atrium and kidney (data 
not shown). When the level of CGRP mRNA in SHR was compared with that of WKY as the ratio of CGRP mRNA to GAPDH mRNA, a significant decrease was found in SHR, as shown in Fig. 2B.

As shown in Fig. 2A and B, long-term treatment with L158,809 , olmesartan or temocapril caused a significant increase in the levels of CGRP mRNA in DRG of SHR. However, hydralazine treatment had no effect on the CGRP mRNA levels.

\section{Changes in Tissue CGRP Contents}

As shown in Fig. 3, in both control SHR and WKY, the level of CGRP-LI in the mesenteric artery (Fig. 3A) was about 10 -fold greater than those in the atrium (Fig. 3B). Both the mesenteric artery and the atrium in control SHR at 15 weeks of age had greater levels of CGRP-LI than those in age-matched WKY. A significant difference between SHR and WKY in CGRP-LI level was found in both tissues (Fig. 3).

Long-term treatment with L-158,809, olmesartan or temocapril caused significantly greater increases in the contents of CGRP-LI in the mesenteric artery than control SHR (Fig. 3A). Small but significant increases in the CGRP-LI level were found in the atrium treated with temocapril, L-158,809 and olmesartan (Fig. 3B). However, hydralazine treatment was without effect on CGRP-LI level in either tissue.

\section{Discussion}

The present findings demonstrated that the amount of CGRP mRNA in DRG of SHR at 15 weeks of age was smaller than that in age-matched WKY, and long-term treatment with an ACE inhibitor and $\mathrm{AT}_{1} \mathrm{R}$ antagonist restored the reduced levels of CGRP mRNA in SHR to levels similar to those in WKY. We showed in a previous study that the decrease in the CGRP mRNA level of SHR was age-related, but that in WKY it did not change (21). The amount of CGRP mRNA in the DRG of SHR at 12 weeks of age has also been reported to be decreased when compared with age-matched WKY (22). In addition, the content of CGRP in the dorsal horn of the spinal cord has been reported to be lower in SHR at 12-14 weeks of age than in age-matched WKY (23). In in vivo studies, we previously reported that electrical stimulation of the lower thoracic spinal cord (Th9-12) in the pithed rat produces a frequency-dependent depressor response, which is abolished by the neurotoxin, tetrodotoxin, or by the CGRP receptor antagonist, $\operatorname{CGRP}(8-37)$, indicating that the response is mediated by endogenous CGRP released from the CGRPergic nerves (24). In a recent study, the depressor response to spinal cord stimulation in pithed SHR at 15 weeks of age was significantly smaller than that in agematched WKY (13). Thus, it is very likely that the outflow of CGRPergic vasodilator nerves from the spinal cord to peripheral blood vessels via the dorsal roots decreases in SHR.
In in vitro studies, we previously reported that CGRPergic nerve-mediated vasodilation in response to periarterial nerve stimulation of the mesenteric artery in SHR was smaller than that in WKY, and the decrease in the response was age-related (12). In addition, the neurogenic release of CGRP-LI in the mesenteric artery of SHR at 15 weeks of age has been shown to be smaller than that in age-matched WKY $(12,13)$. The present study demonstrated that the content of CGRP-LI in the mesenteric artery of SHR was greater than that in WKY. This finding suggests that the increase in CGRP-LI level in SHR is due to the reduced release of CGRP from CGRPergic nerve terminals, even though the levels of CGRP mRNA in SHR decreased. Since CGRPergic nerves have been shown to inhibit the function of adrenergic nerves (3), the decreased function of CGRPergic nerves in SHR enhances the sympathetic adrenergic vasoconstriction. A recent study demonstrated that $\alpha$-CGRP/calcitonin gene knockout mice displayed an elevated mean blood pressure compared with wild-type mice, indicating that $\alpha$-CGRP is involved in the long-term regulation of resting blood pressure (25). Therefore, a reduction in the potent vasodilator CGRP may contribute to the increase in vascular resistance and blood pressure in SHR.

In the present study, the amount of CGRP mRNA in DRG of SHR was significantly increased by long-term treatment with $\mathrm{AT}_{1} \mathrm{R}$ antagonists (L-158,809, olmesartan) or ACE inhibitor (temocapril), whereas the vasodilator (hydralazine) treatment did not show such an effect. This finding suggests that lowered blood pressure is not responsible for the increased levels of CGRP mRNA. In in vitro studies, we have previously shown that the reduced vasodilation mediated by CGRPergic nerves in SHR mesenteric arteries is restored by long-term treatment with an ACE inhibitor, such as captopril or temocapril $(16,17)$. However, there was no such effect after treatment with either hydralazine, $\beta$-adrenoceptor antagonists (propranolol and pindolol), or calcium antagonists (nicardipine, amlodipine and pranidipine), despite the reduction in mean blood pressure by these agents $(16,17)$. Direct application of ACE inhibitors to the mesenteric artery of non-treated SHR had no effect on the CGRPergic nervemeditated vasodilation (17), indicating that long-term application is responsible for the improvement of CGRPergic function. In addition, an in vivo study demonstrated that long-term treatment of SHR with the ACE inhibitor, captopril, lowered the blood pressure and restored the reduced depressor response induced by spinal cord stimulation (13). We previously reported that angiotensin II as well as angiotensin I and renin substrate ( $N$-acetyltetradecapeptide) inhibited the neurotransmission of CGRPergic nerves in SHR but not WKY (15). Thus, it is very likely that long-term inhibition of the renin-angiotensin system by the ACE inhibitor improves the decreased CGRPergic nerve function in SHR. Therefore, it is suggested that circulatory angiotensin II as well as the converted one in the vasculature is responsible for the decreased function of CGRPergic nerves in SHR. In the DRG, 
stimulation of $\mathrm{AT}_{1} \mathrm{R}$ has been shown to inhibit synthesis of CGRP (26). Deng et al. (27) reported the increased level of angiotensin II in the heart of 15 week-old SHR than agematched WKY, suggesting that the angiotensin II level may increase in DRG of SHR. Therefore, it is more likely that increased circulatory or tissue production of angiotensin II is responsible for the reduced function of CGRPergic nerves in SHR.

The present study showed that the contents of CGRP in the mesenteric artery and atrium of SHR were further increased by long-term treatment with $\mathrm{AT}_{1} \mathrm{R}$ antagonists and ACE inhibitor but not with hydralazine. It was found that the increase in CGRP contents was much greater in the mesenteric artery than the atrium. This finding suggests that CGRPergic nerves play a major role in the control of the mesenteric arterial tone. In a previous study, long-term treatment with ACE inhibitor was shown to facilitate both the neurogenic release of CGRP and CGRPergic nerve-mediated vasodilation in the mesenteric artery of SHR (17). Since angiotensin II presynaptically attenuated the neurotransmission of CGRPergic nerves in SHR (15), the inhibition of angiotensin II action may facilitate the neurotransmission. In addition, the increase in CGRP contents of the mesenteric artery and synthesis of CGRP in DRG after treatment with $\mathrm{AT}_{1} \mathrm{R}$ antagonist and ACE inhibitor further facilitate the neurotransmission of CGRPergic nerves in SHR. Since CGRP is the most potent vasodilator known $(1,28)$, it is possible that the increased release of CGRP from the nerve may induce the decrease in the vascular resistance and lead to a reduction in blood pressure.

In conclusion, the present study suggests that $\mathrm{AT}_{1} \mathrm{R}$ antagonist and ACE inhibitor have the ability to improve the reduced expression of CGRP mRNA, which may facilitate neurotransmission of CGRPergic nerves in SHR. It is likely that the improved effect of CGRP function by $\mathrm{AT}_{1} \mathrm{R}$ antagonist and ACE inhibitor contributes in part to the hypotensive effect of these drugs.

\section{Acknowledgements}

L-158,809 was generously donated by Merck \& Co. (Rahway, USA), and olmesartan and temocapril were generously donated by Sankyo Co. (Tokyo, Japan).

\section{References}

1. Kawasaki H, Takasaki K, Saito A, Goto K: Calcitonin gene-related peptide acts as a vasodilator neurotransmitter in mesenteric resistance vessels of the rat. Nature 1988; 335: $164-167$.

2. Toda N, Okamura T: Mechanism of neurally induced monkey mesenteric artery relaxation and contraction. Hypertension 1992; 19: 161-166.

3. Kawasaki H, Nuki C, Saito A, Takasaki K: Role of calcitonin gene-related peptide containing nerves in the vascular adrenergic neurotransmission. J Pharmacol Exp Ther 1990;
252: 403-409.

4. Kawasaki H, Nuki C, Saito A, Takasaki K: NPY modulates neurotransmission of CGRP-containing vasodilator nerves in rat mesenteric arteries. Am J Physiol 1991; 261: H683-H690.

5. Yamamoto AY, Toyama M: Calcitonin gene-related peptide in the nervous system. Prog Neurobiol 1989; 33: 335-386.

6. Marti E, Gibson SJ, Polak MN, et al: Ontogeny of peptideand amine-containing neurons in motor, sensory, and autonomic regions of rat and human spinal cord, dorsal root ganglia and rat skin. J Comp Neurol 1987; 266: 332-359.

7. Folkow B, Hallback M, Lundgren Y, Weiss L: Structurally based increase in flow resistance in spontaneously hypertensive rats. Acta Physiol Scand 1970; 79: 373-378.

8. Zimmerman BG: Peripheral neurogenic factors in acute and chronic alterations of arterial pressure. Circ Res 1983; 53: 121-130.

9. Head RJ: Hypernoradrenergic innervation: its relationship to functional and hyperplastic changes in the vascular of the spontaneously hypertensive rat. Blood Vessels 1989; 26: $1-20$.

10. Kawasaki H, Cline WH Jr, Su C: Enhanced angiotensinmediated facilitation of adrenergic neurotransmission in spontaneously hypertensive rats. J Pharmacol Exp Ther 1982; 221: 112-116.

11. Kawasaki H, Cline WH Jr, Su C: Involvement of the vascular renin-angiotensin system in beta adrenergic receptormediated facilitation of vascular neurotransmission in spontaneously hypertensive rats. J Pharmacol Exp Ther 1984; 231: 23-32.

12. Kawasaki H, Saito A, Takasaki K: Age-related decrease of calcitonin gene-related peptide-containing vasodilator innervation in the mesenteric resistance vessel of the spontaneously hypertensive rat. Circ Res 1990; 67: 733-743.

13. Kawasaki H, Nuki Y, Yamaga N, Kurosaki Y, Taguchi T: Decreased depressor response mediated by calcitonin generelated peptide (CGRP)-containing vasodilator nerves to spinal cord stimulation and levels of CGRP mRNA of the dorsal root ganglia in spontaneously hypertensive rats. $\mathrm{Hy}$ pertens Res 2001; 23: 693-699.

14. Kawasaki H, Takasaki K: Age-related decrease of neurogenic release of calcitonin gene-related peptide from perivascular nerves in spontaneously hypertensive rats. Clin Exp Hypertens 1992; A14: 989-1001.

15. Kawasaki H, Takenaga M, Araki H, Futagami K, Gomita $\mathrm{Y}$ : Angiotensin inhibits neurotransmission of calcitonin gene-related peptide-containing vasodilator nerves in mesenteric artery of spontaneously hypertensive rats. $J$ Pharmacol Exp Ther 1998; 284: 508-515.

16. Kawasaki H: Effects of chronic administration of antihypertensive drugs on vasodilation mediated by calcitonin gene-related peptide-containing vasodilator nerves in spontaneously hypertensive rats. Clin Exp Pharmacol Physiol 1992; 19: 569-573.

17. Kawasaki H, Okazaki M, Nakatsuma A, Mimaki Y, Araki H, Gomita Y: Long-term treatment with angiotensin converting enzyme inhibitor restores reduced calcitonin generelated peptide-containing vasodilator nerve function in mesenteric artery of spontaneously hypertensive rats. Jpn J Pharmacol 1999; 79: 221-229.

18. Siegl PKS, Chang RSL, Mantlo NB, et al: In vivo Pharma- 
cology of L-158,809, a new highly potent and selective nonpeptide angiotensin II receptor antagonist. $J$ Pharmacol Exp Ther 1992; 262: 139-262.

19. Mizuno M, Sada M, Ikeda M, et al: Pharmacology of CS866, a novel nonpeptide angiotensin II receptor antagonist. Eur J Pharmacol 1995; 285: 181-188.

20. Chomczynski P, Sacchi N: Single-step method of RNA isolation by acid guanidinium thiocyanate-phenol-chloroform extraction. Anal Biochem 1987; 162: 156-159.

21. Yamaga N, Kawasaki H, Inaizumi K, Shimizu M, Nakamura A, Kurosaki Y: Age-related decrease in calcitonin gene-related peptide mRNA in the dorsal root ganglia of spontaneously hypertensive rats. Jpn J Pharmacol 2001; 86: 448-450.

22. Supowit SC, Ramana CV, Westlund KN, Dipette DJ: Calcitonin gene-related peptide gene expression in the spontaneously hypertensive rat. Hypertension 1993; 21: 1010-1014.

23. Westlund KN, Dipette DJ, Carson K, Holland OB: Decreased spinal cord content of calcitonin gene-related peptide gene in the spontaneously hypertensive rat. Neurosci Lett 1991; 131: 183-186.
24. Taguchi T, Kawasaki H, Imamura T, Takasaki K: Endogenous calcitonin gene-related peptide mediates nonadrenergic noncholinergic depressor response to spinal cord stimulation in the pithed rat. Circ Res 1992; 71: 357-364.

25. Gangula PR, Zhao H, Supowit SC, et al: Increased blood pressure in alpha-calcitonin gene-related peptide / calcitonin gene knockout mice. Hypertension 2000; 35: 470-475.

26. Li J, Zhao H, Dipette DJ, Supowit SC, Wang DH: Reciprocal role of the $\mathrm{AT}_{1}$ receptor in modulating renal and neuronal AT 1 mRNA expression. J Am Soc Nephrol 1999; 10 (Suppl 11): S18-S22.

27. Deng A, Zheng D, Wang B, et al: The role of the renin-angiotensin and cardiac sympathetic nervous system in the development of hypertension and left ventricular hypertrophy in spontaneously hypertensive rats. Hypertens Res 1999; 22: 217-221.

28. Brain SD, Williams TJ, Tippins JR, Morris HR, MacIntyre I: Calcitonin gene-relates peptide is a potent vasodilator. Nature 1985; 313: 54-56. 